\title{
Combination of XANES spectroscopy and molecular dynamics to probe the local structure in disordered systems
}

\author{
Patrick J. Merkling, ${ }^{1}$ Adela Muñoz-Páez, ${ }^{2}$ Rafael R. Pappalardo, ${ }^{1}$ and Enrique Sánchez Marcos ${ }^{1, *}$ \\ ${ }^{1}$ Departamento de Química Física, Universidad de Sevilla, 41012 Sevilla, Spain \\ ${ }^{2}$ Departamento de Química Inorgánica, ICMSE Universidad de Sevilla, CSIC, 41012 Sevilla, Spain
}

(Received 8 May 2001; published 7 August 2001)

\begin{abstract}
Individual configurations obtained from molecular dynamics have been combined with the computation of $\mathrm{x}$-ray absorption near-edge structure (XANES) spectra to obtain a theoretical estimation of the spectrum corresponding to a system in a condensed medium lacking long-range order. The influence of the different geometries on the spectrum is studied. The results obtained indicate that the reproduction of the features of the XANES spectrum requires a good sampling of geometrical arrangements. As a test case, an aqueous solution of $\mathrm{Cr}\left(\mathrm{H}_{2} \mathrm{O}\right)_{6}^{3+}$ was selected, since its simulation reproduces well structural results. The contribution of the second hydration shell on the shape of the spectrum was determined.
\end{abstract}

DOI: 10.1103/PhysRevB.64.092201

PACS number(s): 61.10.Ht, 61.20.Ja

\section{INTRODUCTION}

X-ray-absorption spectra (XAS) have been typically split into an energy region close to the edge, termed $\mathrm{X}$-rayabsorption near-edge structure or XANES, and a region above the edge, which is called extended $\mathrm{X}$-ray-absorption fine structure (EXAFS). The XANES spectrum is known to be sensitive to both geometrical properties, such as local symmetry around the absorber atom and electronic properties, such as the formal oxidation state, but unlike for the EXAFS region, ${ }^{1}$ at present no broadly accepted theory has been developed to establish a direct relationship between structure and spectrum. Over the last years, efforts have been undertaken to understand the relationship between XANES spectra and geometry around the absorber atom. ${ }^{2-4}$ A large number of papers in the fields of physics, chemistry, and materials science have used these spectra as a fingerprint or to establish qualitative correlations between a given feature and a structural parameter. ${ }^{5}$ Nevertheless the modeling of XANES spectra in condensed matter lacking long-range order, such as biological media, ionic solutions, glasses, supercritical fluids, or three-way catalysts, has usually been quite crude due to several approximations. On one hand, studies around absorber atoms usually consider either the environment formed by the first coordination shell or an approximately regular arrangement of neighbors. Usually, the latter has been achieved by building clusters of growing size using crystallographic parameters, quantum-chemical optimizations, or a variety of other strategies. ${ }^{6-8}$ But shells beyond the first one are not accounted for realistically in systems without long-range order. On the other hand, discrepancies can be due to some of the approximations in the quantumchemical calculations such as the use of muffin-tin potentials, self-energy approximations, self-consistency, .... In this field sensible progress has been registered over the last years, ${ }^{9,10}$ and computer programs are becoming readily accessible. For all these reasons, the matching of XANES experimental spectra in a disordered phase is a stimulating challenge whose resolution may provide useful information not accessible by other means.
Molecular-dynamics (MD) configurations might provide an ideal source of XAS independent structural information. Although in several articles EXAFS spectra have been generated based on MD structural data, ${ }^{11,12}$ only a preliminary investigation in the XANES region has been published, to the best of our knowledge. ${ }^{13} \mathrm{Up}$ to now, the calculation of these spectra was based on the assumption of a single structure. The purpose of this work is to evaluate the practical influence of the distribution of geometries on a model spectrum and to show that the use of MD can lead to excellent theoretical spectra. As a representative example of a disordered system, a $\mathrm{Cr}^{3+}$-containing aqueous solution was chosen. It is well studied by many techniques and its intrinsic simplicity makes it a good candidate. ${ }^{14,15}$ XANES spectra of the $\mathrm{Cr} K$-edge are calculated by a combination of statistical and quantum-mechanical calculations. Structures are obtained from MD simulations that use a specially designed $\left[\mathrm{Cr}\left(\mathrm{H}_{2} \mathrm{O}\right)_{6}\right]^{3+}-\mathrm{H}_{2} \mathrm{O}$ intermolecular potential, which is able to deal with the important many-body terms present in the strong ion-solvent interactions. ${ }^{16}$ Simulations using this potential have already demonstrated convincing agreement with experimental structural, energetic, dynamical, and spectroscopic results. ${ }^{17,18}$ The structures are input in the FEFF program, ${ }^{19}$ the code to calculate XAS spectra. Results are compared with an experimental XANES spectrum of a dilute aqueous $\mathrm{Cr}\left(\mathrm{NO}_{3}\right)_{3}$ solution recorded as described elsewhere. $^{21,20}$

\section{METHODS}

MD simulations of a system consisting of $1 \mathrm{Cr}^{3+}$ $+6\left(\mathrm{H}_{2} \mathrm{O}\right)_{I}+512\left(\mathrm{H}_{2} \mathrm{O}\right)_{\text {bulk }}$ were generated using an experimental version (3.0) of the MOLDY program. ${ }^{22} \mathrm{~A}$ flexible hydrated ion model was employed such that $\mathrm{Cr}^{3+}-\left(\mathrm{H}_{2} \mathrm{O}\right)_{I}$ interactions were described by an effective ion-water first-shell potential including many-body interactions of the whole first hydration shell, ${ }^{17}$ whereas $\left[\mathrm{Cr}\left(\mathrm{H}_{2} \mathrm{O}\right)_{6}\right]^{3+}-\left(\mathrm{H}_{2} \mathrm{O}\right)_{\text {bulk }}$ interactions were described by the hydrated ion-water potential. ${ }^{16}$ Bulk water-water interactions were described by the TIP4P potential. A 1-ns simulation time was generated. These microcanonical simulations used periodic boundary conditions 
and an Ewald summation for Coulombic interactions. The newly implemented symplectic integration algorithm of the leapfrog type in the MOLDY program allowed for an increased time step of 2.0 fs. The system was thermalized at $298 \mathrm{~K}$.

A total of 100 snapshots taken every 10 ps was used for XANES calculations of instantaneous spectra and averaged. Calculations of the XANES spectra were carried out by means of version 8.10 of the FEFF program. It was found that the inclusion of hydrogen atoms was necessary for obtaining a realistic potential but leads to a sensible degradation of the spectrum when included as backscatterers. For this reason, they were not included in the full multiple-scattering (FMS) part of the calculation. No adjustable parameter was introduced in the calculation. In the computations presented, two kinds of oxygen atoms were distinguished, the more perturbed first-shell ones, whose treatment needed to be extended up to an angular moment of $l=3$, and other oxygen atoms with $l=2$, while one type of hydrogen atom was used. This procedure was a way to introduce the hydrated ion concept within the theoretical computations of the XAS spectra.

Test calculations have revealed that the spectrum is almost unmodified if the selected snapshots are limited by a cutoff centered around the absorber atom above $6.0 \AA$. Since computation time was not a major concern, a safe cutoff of $7.0 \AA$ for oxygen atoms was chosen. A radius of $4.5 \AA$ was chosen for the self-consistent field potential and for the FMS paths, ensuring that second-shell oxygen atoms were being considered in the calculation of the spectrum. A "muffin-tin" potential was applied with an overlapping factor according to Norman's criterion of $15 \%$. The broadening of the spectrum was set to a minimum $(-1 \mathrm{eV})$. A noticeable improvement in the ability to reproduce the experimental XANES spectrum is observed when using FEFF 8.10 as compared to the spectra computed with previous versions.

\section{RESULTS AND DISCUSSION}

The averaged XANES theoretical spectrum, using the Hedin-Lundqvist approximation, ${ }^{25}$ is compared to the experimental one in Fig. 1. The overall agreement of the two spectra is excellent. First of all, the edge position and intensity were reproduced accurately, and the "shoulder" (around $6020 \mathrm{eV}$ ) was also well reproduced, both in position and intensity, although the intensity ratio edge/shoulder was lower than in the experimental case. The hump in the 6050 $\mathrm{eV}$ region and the oscillations between 6040 and $6100 \mathrm{eV}$ were in good agreement as well. Together with a previous EXAFS study that reproduced the experimental spectrum, ${ }^{23}$ this study confirms that the structural data from our MD simulation are fully consistent with XAS spectra. These structural data are expressed more accurately in terms of radial distribution functions $g(r)$ given in Ref. 17, but it shall suffice to say that the first-shell coordination number around the $\mathrm{Cr}^{3+}$ is 6 , at an average distance of $2.0 \AA$, whereas the corresponding values for the second shell are 14 and $4.08 \AA$, respectively.

Since the role played by hydrogen atoms in the determination of XAS spectra is not well established, ${ }^{2,3,24}$ a tentative

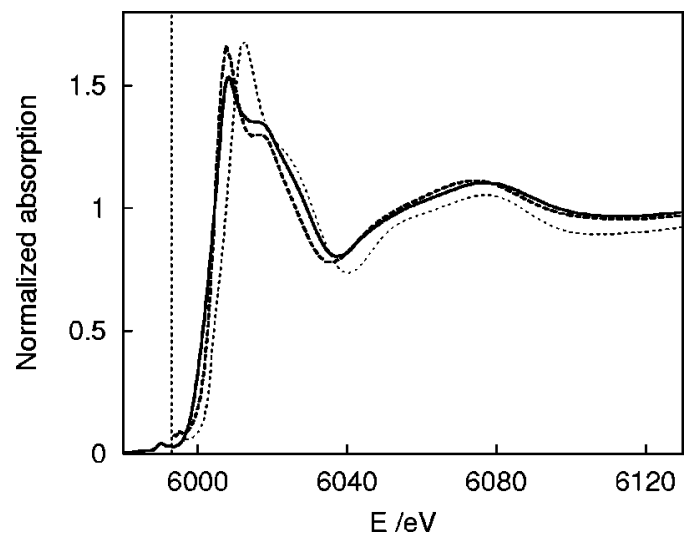

FIG. 1. Comparison of XANES spectra from experiment (bold solid line) and from the MD simulation average (bold dashed line). The dotted curve corresponds to the MD simulation average excluding hydrogen atoms from the calculations. The Fermi level at 5993 $\mathrm{eV}$ for the theoretical calculations (including hydrogen atoms) is indicated.

theoretical XANES spectrum has been computed over the same set of snapshots without including hydrogen atoms. The resulting spectrum is shown in Fig. 1 (dotted line). As a consequence of a shift in the Fermi level of $+3 \mathrm{eV}$, a global displacement of the curve is obtained. This effect results from the elimination of electropositive atoms in the selfconsistent computation of the wave function. Apart from the global displacement, the edge is substantially broadened and masks the characteristic shoulder at around $6020 \mathrm{eV}$; the intensity of the oscillation in the region $6040-6100 \mathrm{eV}$ is reduced.

In Fig. 2(a), the averaged spectrum discussed previously is represented in comparison with two limiting lines corresponding to the standard deviation of individual configuration contributions to the spectrum. It is worth noting that the absolute indetermination is large. This contrasts with the traditional view that structural disorder, typically expressed through Debye-Waller factors, can be neglected in XANES spectra since they correspond to low $k$. The indetermination is most important in the region of high slope, and interestingly, it is also high in the region of the shoulder. In Fig. 2(b), XANES spectra of individual instantaneous structures of the simulation are displayed. The interesting fact derived from this figure is that most spectra are in fair agreement with the averaged one over some regions, but they are quite different over other energy regions. The edge can be slightly displaced and the feature corresponding to the shoulder in the averaged spectrum shows different shapes ranging from a narrow shoulder to a new peak. This explains the importance of making a thorough sampling of the configurational space. The damping, included softly by the consideration of a large number of individual computed spectra, converts peaks and shoulders into the characteristic features observed in the experimental spectrum. Considering the magnitude of the standard deviations and the peculiar shape of the spectrum, it seems unlikely that it can be modeled by a single representative configuration.

To identify the contributions responsible for each feature 

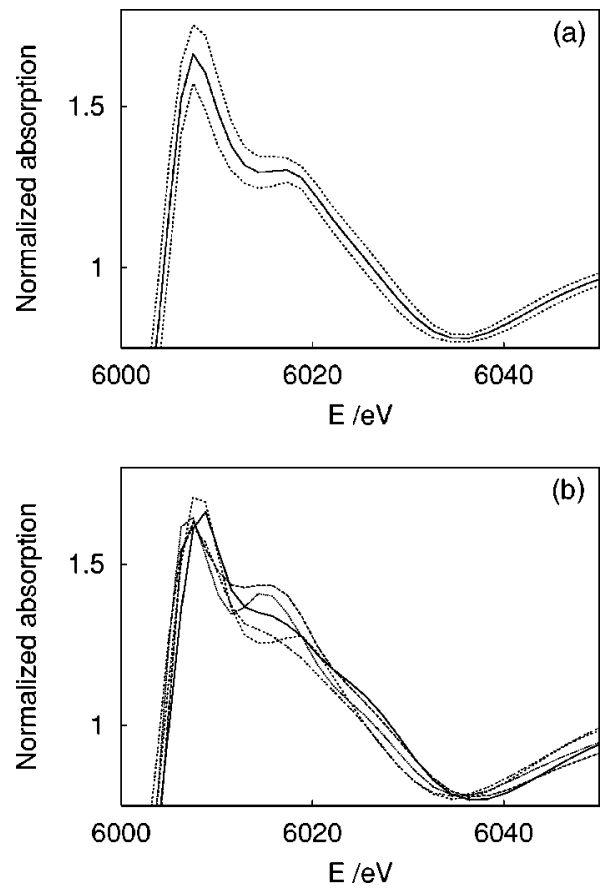

FIG. 2. Calculated XANES spectra: (a) Averaged spectrum (solid line) and its standard deviation (dotted lines) and (b) individual XANES spectra computed from different snapshots.

of this spectrum, calculated spectra of model structures are represented in Fig. 3, vertically offset by 0.5 from each other. In a study on $\mathrm{Ni}^{2+}$ hydration, Benfatto et al. ${ }^{2}$ make calculations of first-shell-only clusters \{i.e., $\left.\left[\mathrm{Ni}\left(\mathrm{H}_{2} \mathrm{O}\right)_{6}\right]^{2+}\right\}$ and find a XANES spectrum that exhibits a small shoulder. When the orientation of the first-shell water molecules is modified, this shoulder rises. Following this reasoning line, we performed three calculations: (i) the average from MD simulation snapshots when considering only first-shell water molecules, (ii) a symmetric cluster that includes only the first coordination shell water molecules, and (iii) a first- and second-shell symmetrical cluster. The distances in the symmetric clusters were chosen using the radial distribution functions of the simulation. The geometry is such that every first-shell hydrogen atom is hydrogen bonded linearly to a second-shell oxygen atom. Twelve second-shell water molecules are thus present in this cluster.

The averaged spectrum corresponding to first-shell water molecules using the snapshots from MD simulations (line 1 in Fig. 3) is quite instructive. With respect to the spectrum of a symmetric first-shell cluster (line 2), differences are quite small. The main difference is that the edge intensity is lowered whereas the shoulder has the same intensity and becomes marginally broader. It can also be seen that at higher energies, the oscillations are smeared out. In the comparison of the spectra from MD simulations where only one (line 1) or several shells (bold dashed line in Fig. 1) are considered, it appears that the differences are important. This is also the case when the spectrum corresponding to the symmetric cluster with only one hydration shell is compared with the one having two hydration shells (line 3). Thus, when secondshell contributions are included in potential and full

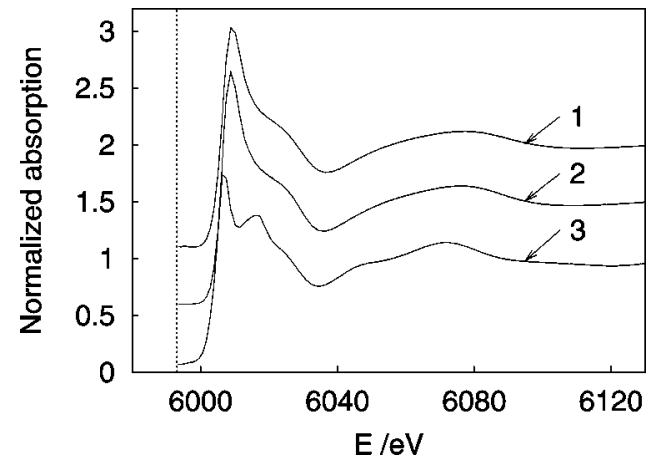

FIG. 3. Calculated XANES spectra corresponding, from top to bottom, to an averaged spectrum from MD snapshots of first-shell environment (1), a symmetrical first-shell cluster (2), and a symmetrical cluster of first- and second-shell water molecules (3).

multiple-scattering calculations, the shape of the spectrum is much more similar to the experimental one. The symmetric cluster reproduces several of the features of the spectrum, although at the position of the shoulder a new peak appears. One can imagine that the final appearance of the shoulder at $6020 \mathrm{eV}$ in the calculated (and experimental) XANES spectrum is the result of the statistical average introduced by the set of MD snapshots used to compute the individual spectra. In this sense, new efforts should be undertaken to relate sets of MD configurations exhibiting common geometrical properties (characterized by $n$-body distribution functions) with structural features of the absorption spectrum. This would allow a more precise interpretation of the experimental spectrum.

Summarizing, the calculated XANES spectrum reproduces perfectly the experimental one, leading us to think that both the structural data obtained in the simulation and the algorithm implemented in FEFF are reliable. A study of the cluster showed that important contributions to the spectrum are due to the second-shell water molecules. The correct smearing out and quantitative agreement with the experimental spectrum are only possible if one includes the disorder of the liquid phase, for example in a statistical way as was done in this study. The importance of fluctuations of the structure around absorber atoms forces one to take them into account when establishing a correlation between features of the XANES spectrum and structural parameters. Whereas in EXAFS a model (or average) structure can be used taking into account the disorder with Debye-Waller factors, the results presented here indicate that this approach doesn't seem to be valid for the XANES region of the spectrum.

\section{ACKNOWLEDGMENTS}

P. J. Merkling is grateful to the Deutsche Forschungsgemeinschaft for financial support. We thank Professor J. J. Rehr for his expert help and suggestions and Dr. K. Refson for supplying us with the latest version of the MOLDY program. We acknowledge the Spanish DGICYT (IFD97-1188) for financial support. 
*Email address: sanchez@simulux.us.es

${ }^{1}$ D. E. Sayers, E. A. Stern, and F. W. Lytle, Phys. Rev. Lett. 27, 1204 (1971).

${ }^{2}$ M. Benfatto, J. A. Solera, J. Chaboy, M. G. Proietti, and J. García, Phys. Rev. B 56, 2447 (1997).

${ }^{3}$ A. L. Ankudinov, S. D. Conradson, J. Mustre de Leon, and J. J. Rehr, Phys. Rev. B 57, 7518 (1998).

${ }^{4}$ S. Díaz-Moreno, A. Muñoz-Páez, and J. Chaboy, J. Phys. Chem. A 104, 1278 (2000).

${ }^{5}$ J. G. Chen, Surf. Sci. Rep. 30, 1 (1997).

${ }^{6}$ A. Muñoz-Páez, S. Díaz-Moreno, E. Sánchez Marcos, and J. J. Rehr, Inorg. Chem. 39, 3784 (2000).

${ }^{7}$ C. Maurizio, F. d'Acapito, M. Benfatto, S. Mobilio, E. Cattaruzza, and F. Gonella, Eur. Phys. J. B 14, 211 (2000).

${ }^{8}$ F. Farges, G. E. Brown, Jr., J. J. Rehr, and S. D. Conradson, Phys. Rev. B 56, 1809 (1997).

${ }^{9}$ A. Ankudinov, J. Synchrotron Radiat. 6, 236 (1999).

${ }^{10}$ Y. Joly, D. Cabaret, H. Renevier, and C. R. Natoli, Phys. Rev. Lett. 82, 2398 (1999).

${ }^{11}$ J. L. Fulton, M. M. Hoffmann, J. G. Darab, B. J. Palmer, and E. A. Stern, J. Phys. Chem. A 104, 11651 (2000).

${ }^{12}$ L. Campbell, J. J. Rehr, G. K. Schenter, M. I. McCarthy, and D. Dixon, J. Synchrotron Radiat. 6, 310 (1999).

${ }^{13}$ A. Pasquarello, I. Petri, P. S. Salmon, O. Parisel, R. Car, E. Tóth, D. Hugh Powell, H. E. Fischer, L. Helm, and A. E. Merbach, Science 291, 856 (2001).

${ }^{14}$ H. Ohtaki and T. Radnai, Chem. Rev. 93, 1157 (1993).

${ }^{15}$ Y. Marcus, Ion Solvation (Wiley, Chichester, 1986).

${ }^{16}$ J. M. Martínez, R. R. Pappalardo, E. Sánchez Marcos, K. Refson,
S. Díaz-Moreno, and A. Muñoz-Páez, J. Phys. Chem. B 102, 3272 (1998).

${ }^{17}$ J. M. Martínez, R. R. Pappalardo, and E. Sánchez Marcos, J. Chem. Phys. 109, 1445 (1998).

${ }^{18}$ J. M. Martínez, R. R. Pappalardo, and E. Sánchez Marcos, J. Am. Chem. Soc. 121, 3175 (1999).

${ }^{19}$ A. Ankudinov, B. Ravel, J. J. Rehr, and S. D. Conradson, Phys. Rev. B 58, 7565 (1998).

${ }^{20}$ H. Sakane, A. Muñoz-Páez, S. Díaz-Moreno, J. M. Martínez, R. R. Pappalardo, and E. Sánchez Marcos, J. Am. Chem. Soc. 120, 10397 (1998).

${ }^{21}$ S. Díaz-Moreno, A. Muñoz-Páez, J. M. Martínez, R. R. Pappalardo, and E. Sánchez Marcos, J. Am. Chem. Soc. 118, 12654 (1996).

${ }^{22}$ K. Refson, Comput. Phys. Commun. 126, 310 (2000).

${ }^{23}$ P. J. Merkling, A. Muñoz-Páez, J. M. Martínez, R. R. Pappalardo, and E. Sánchez Marcos, Phys. Rev. B 64, 012201 (2001).

${ }^{24}$ A. I. Frenkel, G. V. Korshin, and A. Ankudinov, Environ. Sci. Technol. 34, 2138 (2000).

${ }^{25}$ The potential calculations use the Hedin-Lundqvist (H-L) selfenergy approximation. Trials with other approximation methods show no significant improvement. It is worth noting that an approximation formed by the Dirac-Fock self-energy for the core $+\mathrm{H}-\mathrm{L}$ for valence electrons + a constant imaginary part leads to a very similar spectrum. Likewise, the use of a Dirac-Hara + imaginary part of the $\mathrm{H}-\mathrm{L}$ potential induces a shift of $5 \mathrm{eV}$ in the spectrum with respect to the edge, but without changing the shape of the spectrum. 